\title{
Implementação de um sistema on-line para processamento de dados GNSS
}

\author{
Implementation of an on-line system for GNSS data processing \\ Gabriel Diniz de Oliveira ${ }^{1}$ \\ William Rodrigo Dal Poz ${ }^{2}$ \\ Marília Sanglard Almeida ${ }^{3}$
}

Recebido em abril de 2019.

Aprovado em abril de 2019.

\begin{abstract}
RESUMO
O processamento de dados do Global Navigation Satellite System (GNSS) realizado por softwares científicos é uma necessidade crescente nos estudos da Geodésia Espacial. Porém, o manuseio destes, em geral, nem sempre é uma tarefa fácil. Com isso, um sistema que auxilie no processamento é importante para a eficiência das pesquisas de toda comunidade de usuários. Este trabalho aborda a implementação e a validação de um sistema on-line para processamento e análise de dados GNSS, que possibilite o compartilhamento do uso do software Bernese GNSS. O sistema denominado GNSS-UFV está disponível no endereço http://gnss.ufv.br. Este sistema está preparado para recepcionar, auxiliar e gerenciar as solicitações de processamento de dados GNSS nos métodos Posicionamento por Ponto Preciso (PPP) e Relativo no software Bernese GNSS, e posteriormente encaminhar e armazenar os resultados dos processamentos. O PPP do GNSS-UFV foi testado e comparado com o serviço IBGE-PPP. A média das acurácias das resultantes planimétricas foram iguais para os dois sistemas, $0,013 \mathrm{~m}$, já a acurácia média nas componentes altimétricas foi de 0,020m para o IBGE-PPP e 0,018m para o GNSS-UFV. O método Relativo do GNSS-UFV foi comparado com o serviço AUSPOS, onde a média das acurácias das resultantes planimétricas foram de $0,009 \mathrm{~m}$ e $0,010 \mathrm{~m}$ e a média das acurácias das componentes altimétricas foram de $0,021 \mathrm{~m}$ e 0,026m para o AUSPOS e GNSS-UFV, respectivamente.
\end{abstract}

PALAVRAS-CHAVE: Software Bernese GNSS. PPP. Posicionamento Relativo. Serviços on-line GNSS.

\footnotetext{
${ }^{1}$ Universidade Federal de Viçosa - UFV. Programa de Pós-graduação em Eng. Civil, Área de concentração em Informações Espaciais, Instituto Nacional de Colonização e Reforma Agrária INCRA, Brasil. E-mail: gabriel.diniz@ufv.br, gabriel.oliveira@vta.incra.gov.br.

${ }^{2}$ Universidade Federal de Viçosa - UFV. Programa de Pós-graduação em Eng. Civil, Área de concentração em Informações Espaciais, Brasil. E-mail: william.dalpoz@ufv.br ${ }^{3}$ Universidade Federal de Viçosa - UFV. Programa de Pós-graduação em Eng. Civil, Área de concentração em Informações Espaciais, Brasil. E-mail: marilia.almeida@ufv.br
} 


\begin{abstract}
The processing of Global Navigation Satellite System (GNSS) data by scientific software is a growing need in Space Geodesy studies. However, deal with these, in general, is not always an easy task. Thereby, a system that helps in processing is important for researches efficiency of the entire user community. This paper discusses the implementation and validation of an online system for GNSS data processing and analysis, which enables sharing the use of Bernese GNSS software at UFV. The GNSS-UFV system is available at http://gnss.ufv.br. This system is prepared to receive, assist and manage requests for GNSS data processing with Precise Point Position (PPP) and Relative methods by Bernese GNSS software, and then forward and store processing results. The GNSS-UFV PPP was tested and compared with IBGE-PPP service. The average planimetrics resultants accuracy was the same for both systems, $0.013 \mathrm{~m}$, while the average altimetrics components accuracy was $0.020 \mathrm{~m}$ for the IBGE-PPP and $0.018 \mathrm{~m}$ for the GNSS-UFV. The GNSS-UFV Relative method was compared with AUSPOS service, where the average planimetrics resultants accuracy was $0.009 \mathrm{~m}$ and $0.010 \mathrm{~m}$ and the average altimetrics components accuracy was $0.021 \mathrm{~m}$ and $0.026 \mathrm{~m}$ for AUSPOS and GNSS-UFV, respectively.
\end{abstract}

KEYWORDS: Software Bernese GNSS. PPP. Relative Positioning. GNSS on-line services.

\title{
Introdução
}

A disponibilidade de softwares robustos para posicionamento com o Global Navigation Satellite System (GNSS) de alta qualidade tende a ser restrita a produtos comerciais de alto custo, serviços on-line pouco flexíveis, ou a softwares científicos livres, porém, de manuseio complexo.

O setor privado apresenta soluções cada vez mais completas no intuito de disponibilizar ao usuário a capacidade de ter um posicionamento preciso com menor interferência do usuário. Todavia, a concorrência comercial faz com que as empresas desenvolvam programas que não permitem interferência aprofundada nas estratégias de processamento. Além disso, o código-fonte destes programas não são disponibilizados.

A omissão de detalhes no processamento traz insegurança ao usuário a respeito do resultado final alcançado, pois em alguns casos as coordenadas estimadas podem ser precisas, mas não acuradas. Estas omissões do 
processamento e desconfianças nos resultados tendem a inviabilizar pesquisas no meio acadêmico.

Para finalidades científicas como o controle e estimativa de deformação do sistema terrestre, monitoramento de sismos, densificação de redes geodésicas e outras atividades que exigem estimativas precisas de posicionamento e velocidades da área em estudo, o pós-processamento realizado por softwares científicos, torna-se uma alternativa viável aos estudos da Geodésia Espacial (SCARAMBONI e CHAVES, 2013).

Como opção temos o software Bernese GNSS. Este é um software científico e de alta precisão para o processamento de dados GNSS, desenvolvido no Instituto Astronômico da Universidade de Berna na Suíça (FRIDEZ, 2018).

Atualmente algumas instituições internacionais oferecem serviços online para posicionamento com GNSS, porém ainda não foi identificada uma aplicação web que possibilite utilizar como referência os dados das estações da Rede Brasileira de Monitoramento Contínuo dos Sistemas GNSS (RBMC) para o método de posicionamento relativo.

Próximo a isto, tem-se o AUSPOS (JIA, DAWSON e MOORE, 2014), um serviço on-line para processamento de dados GPS disponibilizado pelo Governo da Austrália através da Geoscience Australia. Este serviço utiliza o software Bernese GNSS versão 5.2 para o processamento dos dados pelo método relativo em rede. Usa como referência estações do International GNSS Service (IGS) que possui poucas estações da RBMC inseridas nesta rede.

Entretanto, a seleção das estações de referência no AUPOS é feita automaticamente. Com isso, algumas linhas de base formadas podem atingir centenas de quilômetros, o que pode comprometer o resultado final, uma vez que o Brasil contempla uma das áreas mais complexas da ionosfera (MONICO, 2008). 
Mesmo utilizando linhas de base muito longas para dados coletados no Brasil, o AUSPOS retorna resultados compatíveis com outros serviços de processamento como o IBGE-PPP (ALMEIDA e DAL POZ, 2016).

O IBGE-PPP é um serviço on-line gratuito que utiliza o aplicativo CSRS-PPP (NRCan), sendo disponibilizado pelo IBGE desde abril de 2009 (COSTA et al, 2013).

Similar a esses serviços, este artigo apresenta a implementação de um sistema on-line para processamento e análise de dados GNSS, a partir dos métodos Posicionamento por Ponto Preciso (PPP) e Relativo, o que possibilita o compartilhamento do uso do software Bernese GNSS na UFV.

\section{Sistema GNSS-UFV}

O sistema GNSS-UFV está preparado para recepcionar, auxiliar e gerenciar as solicitações de processamento de dados GNSS nos métodos de PPP e Relativo do software Bernese GNSS, e posteriormente encaminhar e armazenar os resultados dos processamentos.

Detalhes da implementação do sistema podem ser acompanhados no repositório gnss-ufv no GitHub (https://github.com/gabriel-oliveira/gnssufv).

Apesar do software Bernese GNSS possuir uma interface gráfica para auxiliar o usuário nas solicitações de processamento, também é possível realizar solicitações de processamento diretamente por um terminal de comando, utilizando rotinas escritas na linguagem Perl que acompanham o software. Esta possibilidade de solicitação viabiliza a automação dos processamentos por sistemas de terceiros.

Foi criada uma Application Program Interface (API) para fazer uma interface com o software Bernese no uso das rotinas escritas em Perl que executam os módulos do Bernese Process Engine (BPE). A execução destas rotinas presume que os arquivos necessários para o processamento (descritos em Dach et al., 2015) estejam nos respectivos diretórios predefinidos. Sendo 
assim, está API recepciona, busca, verifica e armazena os arquivos necessários para os processamentos antes de iniciá-los.

Outra função fundamental desta API é a criação dos arquivos com informações das estações de rastreio necessários para o processamento de dados GNSS no software Bernese GNSS. Esses arquivos são gerados com base nas informações contidas no cabeçalho dos arquivos RINEX enviados pelos usuários e informações fornecidas pelo formulário de solicitação de processamento da interface web. Estes dados são validados durante a submissão.

Caso os arquivos RINEX não sejam validados uma mensagem de erro é retornada para o usuário na própria interface indicando o(s) erro(s) encontrado(s). Sendo os arquivos validados, as informações do formulário são armazenadas no banco de dados para o processamento.

Este modelo de armazenamento das solicitações de processamento viabiliza o processo de solicitações em paralelo, pois enquanto uma solicitação está sendo processada outras podem estar sendo recebidas pelo sistema.

O software Bernese GNSS integra os seus módulos através de leituras e escritas em arquivos no disco. Esta estrutura inviabiliza a realização de processamentos paralelos pelo mesmo usuário, pois pode-se ocorrer a sobrescrita de arquivos durante os processamentos.

Uma possível opção de processamento paralelo no Windows seria a criação de vários usuários em um mesmo dispositivo, com o encapsulamento da instalação do software para cada usuário. Outra opção seria a instalação do software em diversas máquinas.

\section{Experimentos para validação do sistema}

Esta seção é destinada aos resultados dos experimentos realizados com a finalidade de validação do sistema GNSS-UFV. Para isto, foram processados dados de 24 horas da estação VICO (RBMC), para 365 dias do ano 2018 nos métodos PPP e Relativo. 


\subsection{PPP}

A fim de validar o método PPP do GNSS-UFV os dados processados neste também foram processados no IBGE-PPP. Com isso, os resultados obtidos a partir dos dois sistemas foram comparados por meio de suas acurácias estimadas.

As acurácias foram calculadas conforme descrito em Almeida e Dal Poz (2016), onde suas resultantes planimétricas e componentes altimétricas são estimadas a partir da tendência (discrepância) e precisão (desvio-padrão) das coordenadas estimadas.

Enfatiza-se que as coordenadas de referência utilizadas para o cálculo das discrepâncias foram obtidas a partir da solução multianual SIR17P01 do SIRGAS-CON (SÁNCHEZ, 2017). Estas coordenadas, estão alinhadas ao IGS14 (REBISCHUNG e SCHMID, 2016a) época 2015.0. Em vista disto as coordenadas estimadas (na época de rastreio) foram atualizadas para a época das coordenadas de referência (2015.0), utilizando a velocidade do VEMOS2017 (DREWES e SÁNCHEZ, 2017). Não foi necessário compatibilizar os referenciais, visto que as coordenadas estimadas pelos dois sistemas também são referenciadas ao IGS14.

A Figura 1 ilustra as acurácias das resultantes planimétricas e componentes altimétricas para os sistemas GNSS-UFV e IBGE-PPP. 
Figura 1: Acurácias das resultantes planimétricas (a) e componentes altimétricas (b) do IBGE-PPP e do GNSS-UFV, no método PPP

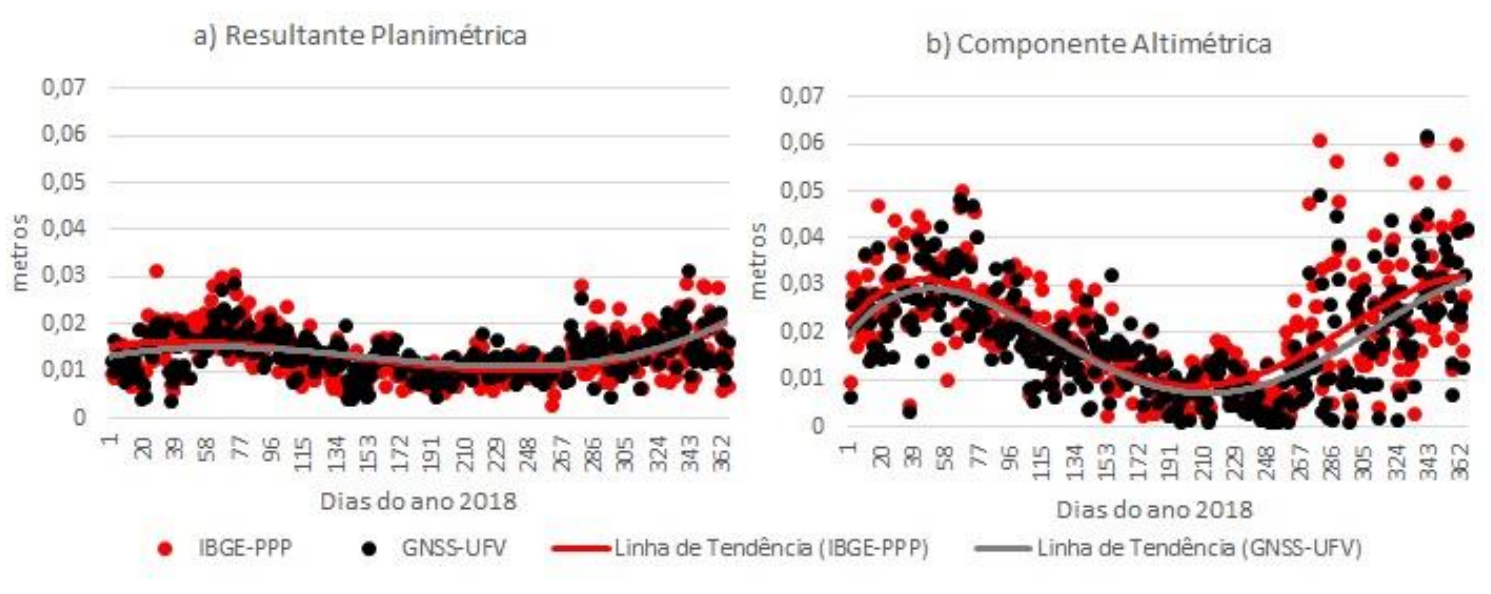

Fonte: Elaborada pelos autores.

Pode-se verificar pela Figura 1 que as acurácias das resultantes planimétricas foram muito próximas para as duas soluções. A média para os dois sistemas foi de $0,013 \mathrm{~m}$. As acurácias das resultantes planimétricas no IBGE-PPP variaram de 0,003m a 0,031m, enquanto no GNSS-UFV variaram de $0,004 \mathrm{~m}$ a $0,031 \mathrm{~m}$.

As acurácias das componentes altimétricas do sistema GNSS-UFV foram menores que do IBGE-PPP. A média das acurácias foram de 0,018m e 0,020m para o GNSS-UFV e IBGE-PPP, respectivamente. Porém, destaca-se que a diferença foi de apenas $0.002 \mathrm{~m}$. Neste caso as acurácias no GNSS-UFV variaram de 0,001m a 0,062m, e no IBGE-PPP variaram de 0,002m a 0,061m.

Este resultado demostra uma confiabilidade nos resultados do sistema GNSS-UFV, entretanto não se pode afirmar que um sistema é melhor que o outro, pois neste cenário de dados com 24 horas de rastreio os sistemas apresentaram compatibilidade dos resultados, porém em cenários diferentes pode não apresentar. 


\subsection{Posicionamento Relativo}

De maneira análoga ao PPP, também foi avaliado o processamento pelo método Relativo do sistema GNSS-UFV, porém desta vez comparado com o serviço AUSPOS.

A rotina de processamento pelo método Relativo do software Bernese GNSS foi alterada para utilizar as estações de referência com coordenadas fixas no ajustamento e com as linhas de base no formato radial, diferente do seu padrão que é do ajustamento livre em rede.

As estações de referência da RBMC utilizadas no sistema GNSS-UFV foram limitadas às três mais próximas (MGBH, CEFE e RJCG) para reduzir o tempo de processamento.

As coordenadas das estações de referência utilizadas no processamento do GNSS-UFV foram coletadas a partir da solução multianual SIR17P01 do SIRGAS-CON (SÁNCHEZ, 2017). Com isso, as coordenadas estimadas da estação rover permanecerão neste mesmo referencial (IGS14, época 2015,0).

De maneira similar ao PPP, as acurácias em resultantes planimétricas e componentes altimétricas foram calculadas conforme descrito por Almeida e Dal Poz (2016). As coordenadas estimadas pelo AUSPOS, por sua vez, são referenciadas ao ITRF2014 na época do rastreio, com isso foi necessária a atualização das mesmas. Neste caso, considera-se a compatibilidade entre o ITRF2014 e o IGS14 (REBISCHUNG e SCHMID, 2016b).

A Figura 2 ilustra as acurácias em resultantes planimétricas e componentes altimétricas, das coordenadas estimadas pelo método Relativo da estação VICO nos sistemas GNSS-UFV e AUSPOS. 
Figura 2: Acurácias em resultantes planimétricas (a) e componentes altimétricas (b) a partir do AUSPOS e do GNSS-UFV, no método Relativo

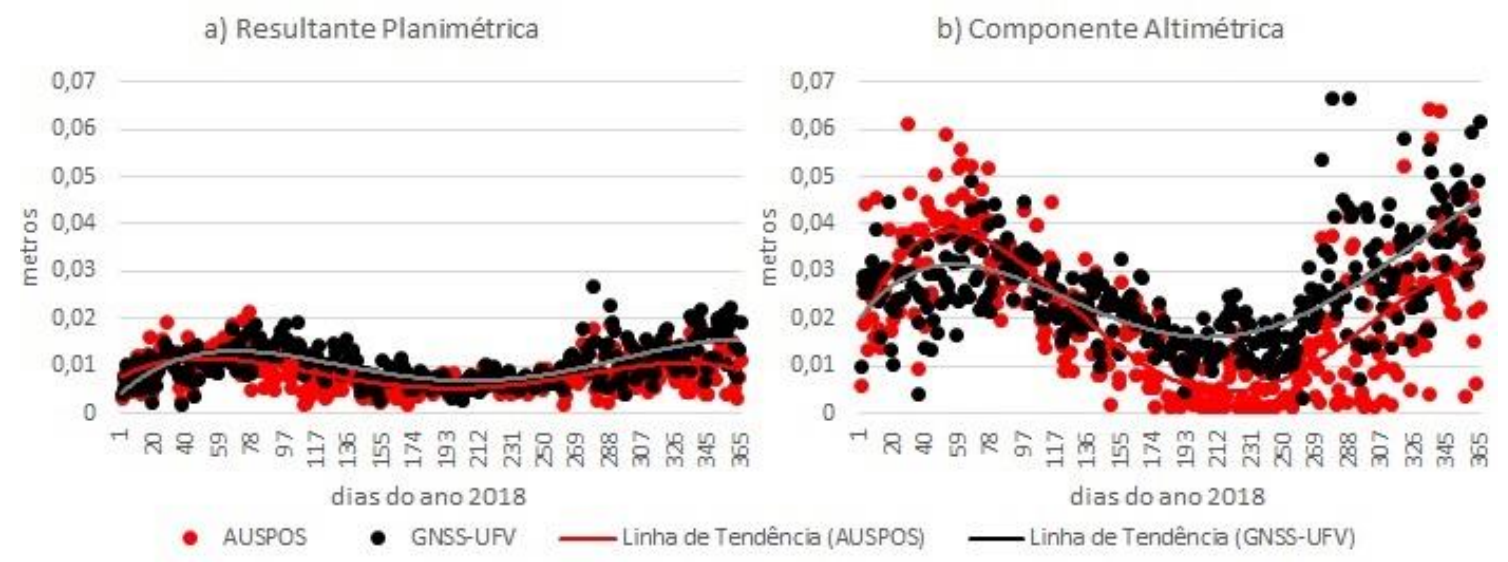

Fonte: Elaborada pelos autores.

As acurácias em resultantes planimétricas ilustradas na Figura 2 variam de $0,002 \mathrm{~m}$ a $0,022 \mathrm{~m}$ com uma média de $0,009 \mathrm{~m}$, para o AUSPOS, e 0,002m a 0,027m com média de 0,010m no GNSS-UFV. Já as acurácias em componentes altimétricas variam de 0,001m a 0,065m com uma média de 0,021m, para o AUSPOS, e 0,004m a 0,076m com média de 0,026m no GNSSUFV.

\section{Considerações Finais, Conclusões e Perspectivas Futuras}

O presente trabalho teve como objetivo apresentar a implementação e validação de uma plataforma on-line para processamento e análise de dados GNSS, que possibilite o compartilhamento do uso do software Bernese GNSS na UFV. Este sistema, denominado GNSS-UFV, se encontra disponibilizado na página http://gnss.ufv.br.

A partir das comparações apresentadas entre o GNSS-UFV, PPP-IBGE e AUSPOS, conclui-se que o sistema é promissor, em função da similaridade dos resultados obtidos em relação aos serviços de posicionamento on-line IBGE-PPP e AUSPOS. 
A licença adquirida do software Bernese GNSS pela UFV é restrita para o uso acadêmico e de pesquisa. Com isso, uma restrição de acesso ao sistema é feita mediante a validação de um cadastro com senha. Esta restrição é feita para garantir o uso adequado da licença disponível.

Tem-se como perspectiva futura o desenvolvimento de aplicação on-line do posicionamento relativo estático rápido. O AUSPOS, por exemplo, não processa dados com intervalos menores do que 60 minutos, impossibilitando seu uso em levantamentos com menores tempo de rastreio. Isso ocorre em função do rigoroso controle de qualidade e deteç̧ão de outliers do software Bernese GNSS. Assim, pretende-se alterar os padrões de controle de qualidade do software, possibilitando seu uso no posicionamento relativo estático rápido.

\section{Agradecimentos}

Esta pesquisa teve o incentivo da Universidade Federal de Viçosa através do Departamento de Engenharia Civil, curso de Pós-Graduação em Engenharia Civil que disponibilizou seus equipamentos, instalações e aplicativos computacionais.

O presente trabalho foi realizado com apoio da Coordenação de Aperfeiçoamento de Pessoal de Nível Superior - Brasil (CAPES) - Código de Financiamento 001.

Agradecimentos ao Instituto Nacional de Colonização e Reforma Agrária (INCRA) pela licença concedida para cursar pós-graduação.

\section{Contribuição dos autores}

A metodologia foi idealizada por todos os autores, sendo implementada pelo primeiro autor, testada e avaliada pelo terceiro autor, sob orientação do segundo autor. Os resultados dos experimentos apresentados neste artigo 
foram executados pelo primeiro e terceiro autor, bem como a redação (textos, revisão bibliográfica, gráficos e figuras), com a orientação e supervisão do segundo autor.

\section{Referências}

ALMEIDA, M. S., DAL POZ, W. R., Posicionamento por Ponto Preciso e Posicionamento Relativo com GNSS: Qual é o método mais acurado atualmente? Boletim de Ciências Geodésicas - Versão On-line, Curitiba, v. 22, n²1, 2016. p.175 -195.

COSTA, S. M. A., SILVA, A. L., LIMA, M. A. A., MOURA JÚNIOR, N.J., QUIRINO, R. A. E ALONSO, P. R. "Serviço online IBGE-PPP: avaliação dos 4 anos de serviço e perspectivas futuras.” Anais do VIII Colóquio Brasileiro De Ciências Geodésicas. Curitiba, 2013.

DACH, R., LUTZ, U., WALSER, S., FRIDEZ, P; Bernese GNSS Software Version 5.2. Manual do Usuário, Astronomical Institute, Universtiy of Bern - AIUB, Bern Open Publishing. DOI: 10.7892/boris.72297; ISBN: 978-3-906813-05-9. Berna, 2015.

DREWES H. e SÁNCHEZ L. Velocity model for SIRGAS 2017: VEMOS2017, Technische Universitaet Muenchen, Deutsches Geodaetisches Forschungsinstitut (DGFI-TUM), IGS RNAAC SIRGAS, supplement to: Drewes H. and Sánchez L. The varying surface kinematics in Latin America: VEMOS 2009, 2015, and 2017, Proceedings of Symposium SIRGAS2017. Mendoza. 2017.

FRIDEZ, P. Bernese GNSS Software. Site <http://www.bernese.unibe.ch>. Acessado em outubro de 2018.

MONICO, J. F. G. Posicionamento pelo GNSS: descrição, fundamentos e aplicações. 2 ed. São Paulo, Unesp, 2008. 480 p.

JIA, M., DAWSON, J. e MOORE, M., AUSPOS: Geoscience Australia's on-line GPS positioning service. Proceedings of the 27th International Technical Meeting of the Satellite Division of the Institute of Navigation (ION GNSS+ 2014). Tampa, 2014. pp. 315-320. 
SCARAMBONI, F. A., CHAVES, J. C., Processamento GNSS no GAMIT/GLOBK utilizando o GridUNESP. Anais do VII Colóquio Brasileiro de Ciências Geodésicas. Curitiba, 2013.

REBISCHUNG, P.; SCHMID, R. IGS14/igs14. atx: a new framework for the IGS products. Proceedings of AGU Fall Meeting 2016. San Francisco, 2016a.

REBISCHUNG, P.; SCHMID, R. Preparations for the IGS realization of ITRF2014. Proceedings of EGU General Assembly. Viena, 2016b.

SÁNCHEZ L., SIRGAS reference frame realization SIR17P01, Technische Universitaet Muenchen, Deutsches Geodaetisches Forschungsinstitut DGFITUM, IGS RNAAC SIRGAS, supplement to: Sánchez L. (2017) Kinematics of the SIRGAS reference frame, Proceedings of Symposium SIRGAS2017. Mendoza, 2017. 